\title{
Coherent Rabi Dynamics of a Superradiant Spin Ensemble in a Microwave Cavity
}

\author{
B. C. Rose, ${ }^{1}$ A. M. Tyryshkin, ${ }^{1}$ H. Riemann, ${ }^{2}$ N. V. Abrosimov, ${ }^{2}$ P. Becker, ${ }^{3}$ H.-J. Pohl, ${ }^{4}$ \\ M. L. W. Thewalt, ${ }^{5}$ K. M. Itoh, ${ }^{6}$ and S. A. Lyon ${ }^{1}$ \\ ${ }^{1}$ Department of Electrical Engineering, Princeton University, Princeton, New Jersey 08544, USA \\ ${ }^{2}$ Leibniz-Institut für Kristallzüchtung, 12489 Berlin, Germany \\ ${ }^{3}$ PTB Braunschweig, 38116 Braunschweig, Germany \\ ${ }^{4}$ VITCON Projectconsult GmbH, 07745 Jena, Germany \\ ${ }^{5}$ Department of Physics, Simon Fraser University, Burnaby, British Columbia, Canada V5A 1S6 \\ ${ }^{6}$ School of Fundamental Science and Technology, Keio University, \\ 3-14-1 Hiyoshi, Kohuku-ku, Yokohama 223-8522, Japan \\ (Received 13 February 2017; revised manuscript received 18 May 2017; published 10 July 2017)
}

\begin{abstract}
We achieve the strong-coupling regime between an ensemble of phosphorus donor spins in a highly enriched ${ }^{28} \mathrm{Si}$ crystal and a 3D dielectric resonator. Spins are polarized beyond Boltzmann equilibrium using spin-selective optical excitation of the no-phonon bound exciton transition resulting in $N=3.6 \times 10^{13}$ unpaired spins in the ensemble. We observe a normal mode splitting of the spin-ensemble-cavity polariton resonances of $2 g \sqrt{N}=580 \mathrm{kHz}$ (where each spin is coupled with strength $g$ ) in a cavity with a quality factor of $75000(\gamma \ll \kappa \approx 60 \mathrm{kHz}$, where $\gamma$ and $\kappa$ are the spin dephasing and cavity loss rates, respectively). The spin ensemble has a long dephasing time $\left(T_{2}^{*}=9 \mu \mathrm{s}\right)$ providing a wide window for viewing the dynamics of the coupled spin-ensemble-cavity system. The free-induction decay shows up to a dozen collapses and revivals revealing a coherent exchange of excitations between the superradiant state of the spin ensemble and the cavity at the rate $g \sqrt{N}$. The ensemble is found to evolve as a single large pseudospin according to the Tavis-Cummings model due to minimal inhomogeneous broadening and uniform spin-cavity coupling. We demonstrate independent control of the total spin and the initial $Z$ projection of the psuedospin using optical excitation and microwave manipulation, respectively. We vary the microwave excitation power to rotate the pseudospin on the Bloch sphere and observe a long delay in the onset of the superradiant emission as the pseudospin approaches full inversion. This delay is accompanied by an abrupt $\pi$-phase shift in the peusdospin microwave emission. The scaling of this delay with the initial angle and the sudden phase shift are explained by the Tavis-Cummings model.
\end{abstract}

DOI: 10.1103/PhysRevX.7.031002

The enhanced collective emission from an ensemble of atomlike systems due to coherent self-stimulated emission was originally described by Dicke [1]. For this phenomenon he coined the term superradiance and showed that the atom ensemble can behave as a large collective pseudospin. Superradiant emission has been observed in numerous physical systems [2-10]. These collective effects are particularly prominent when the coupling between the spin ensemble and the radiation field $(g \sqrt{N}$ for $N$ spins individually coupled with strength $g$ ) is larger than any of the losses in the system $(\kappa+\gamma$, where $\kappa$ is the radiative loss rate and $\gamma$ is the spin dephasing rate). This strong-coupling regime $(g \sqrt{N} \gg \kappa+\gamma)$ has been extensively studied in both theory and experiment [11-14], but clearly resolved dynamics of

Published by the American Physical Society under the terms of the Creative Commons Attribution 4.0 International license. Further distribution of this work must maintain attribution to the author(s) and the published article's title, journal citation, and DOI.
Subject Areas: Mesoscopics, Quantum Physics, Strongly Correlated Materials these collective effects are generally lacking in large ensembles due to strong dephasing (short $T_{2}^{*}=1 / \gamma$ ) $[2,15,16]$. Here, we demonstrate the dynamics of a strongly coupled ensemble of phosphorus donor spins in highly isotopically enriched ${ }^{28} \mathrm{Si}$ with both a long dephasing time $[17,18]$ and uniform coupling to the radiation field (due to the use of a 3D microwave cavity), as shown schematically in Fig. 1(a). For the first time outside of ensembles of Rydberg atoms $[15,16]$, we study a spin-ensemble-cavity system with both of these essential properties allowing for it to be modeled accurately as a single large pseudospin [Fig. 1(b)] offering a simple interpretation of the spin-ensemble-cavity evolution $[1,19]$. In particular, we are able to directly observe the dynamics of superradiant emission under strong excitation, resolving several cycles of coherent excitation transfer between the spin ensemble and the cavity [20]. In contrast to the more extensively studied low excitation limit [21,22], our experiments performed under strong excitation cannot be modeled as a linear system of two coupled harmonic oscillators. Instead, it must be treated with the Tavis-Cummings model [19]. 


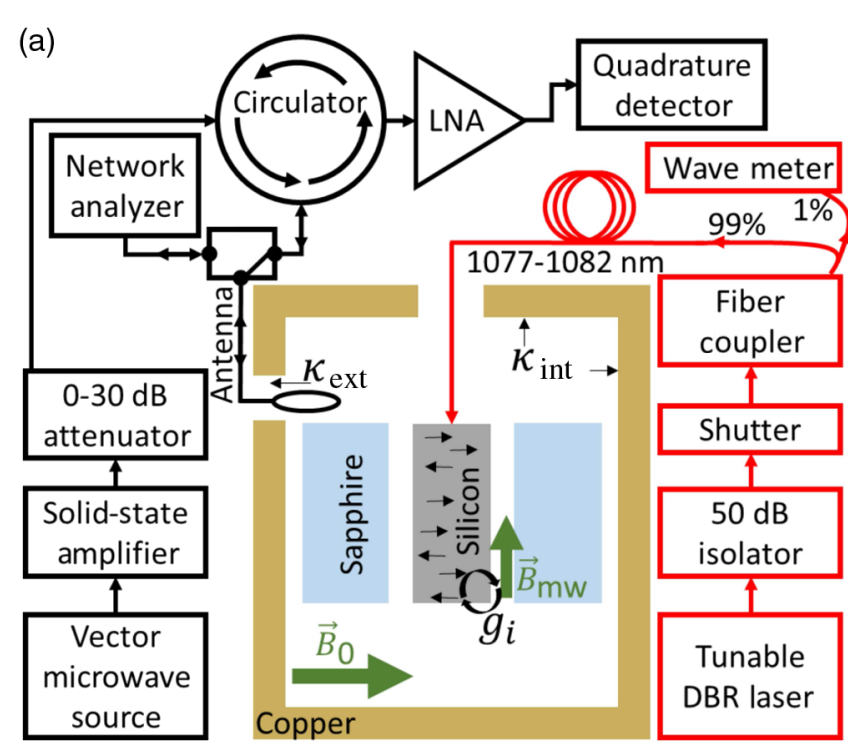

(b)

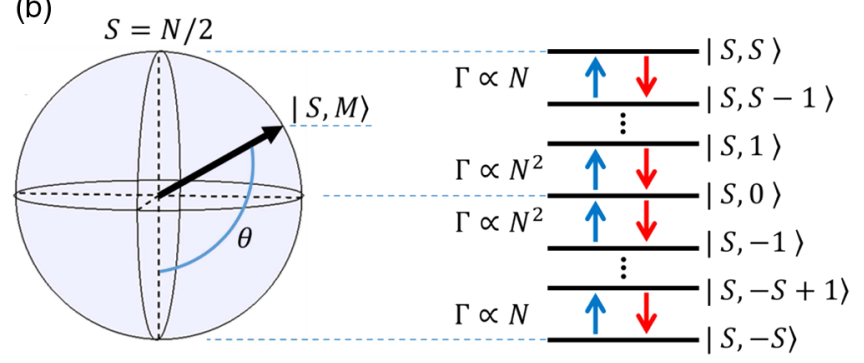

FIG. 1. (a) Experimental scheme for delivering microwave and optical excitation to the phosphorus donor spin ensemble $\left(\hat{s}_{i}, i=1, \ldots, N_{\uparrow}+N_{\downarrow}\right)$ positioned inside a cylindrical dielectric (sapphire) microwave cavity within a helium cryostat $(T=1.5 \mathrm{~K})$. The spins are placed in a $\sim 3500-\mathrm{G}$ dc magnetic field $\left(\vec{B}_{0}\right)$ and coupled magnetically to the cylindrical TE011 cavity mode (microwave magnetic field, $\vec{B}_{\mathrm{mw}}$ ) with individual spin-cavity coupling $g_{i}\left(i=1, \ldots, N_{\uparrow}+N_{\downarrow}\right)$. Current losses in the copper walls $\left(\kappa_{\text {int }}\right)$ and coupling losses $\left(\kappa_{\text {ext }}\right)$ through the antenna along with dephasing in the spin ensemble $\left(\gamma=1 / T_{2}^{*}\right)$ set the maximum window during which the spin-cavity dynamics can be probed. (b) In the case of small inhomogeneous broadening $\left(\omega_{i}=\omega\right)$ and uniform cavity coupling $\left(g_{i}=g\right)$, the spin ensemble can be modeled as a single large pseudospin as shown in the Bloch sphere representation. The sphere surface represents the symmetric superradiant subspace $(|S, M\rangle)$. Strong correlations between spins in these states lead to an enhanced photon emission rate $\Gamma \propto N^{2}$ near the equator $(M=0)$. Uncorrelated spontaneous emission at a rate $\Gamma \propto N$ dominates near the poles $(M= \pm S)$.

Donor electron spins in isotopically enriched ${ }^{28} \mathrm{Si}$ form exceptionally well isolated qubits with the longest electron spin coherence measured in a solid-state environment. The spin decoherence rate $1 / T_{2}$ is less than $1 \mathrm{~Hz}$, and the ensemble dephasing rate $1 / T_{2}^{*}$ can be less than $10 \mathrm{kHz}$ [17]. These rates make the system of interest to the field of quantum computation especially in the context of implementing a long-lived quantum memory. Both rates are 100-1000 times improvement compared to other candidate spin memories [23]. The key figure of merit for implementing a spin-ensemble quantum memory is $g \sqrt{N} T_{2}^{*} \gg 1$, and we demonstrate that we are well beyond satisfying this criterion. Additionally, we achieve relatively uniform spin-cavity coupling with less than 5\% variation across the spin ensemble. Both factors (long $T_{2}^{*}$ and uniform spin-ensemble-cavity coupling) are vital for performing high-fidelity spin-ensemble manipulation while in the strong-coupling regime, which has been an outstanding problem in implementing a spin-ensemble quantum memory [24].

Initialization of the pseudospin is accomplished with a combination of optical excitation, which sets the size of the pseudospin, $S=N / 2$, and microwave manipulation, which sets the initial $Z$ projection of the pseudospin $M$. Previous reports of large superradiant spin ensembles prepared only an initially inverted state $|S, S\rangle[15,16]$, while with our system we are able to prepare any initial pseudospin state $|S, M\rangle$ in the superradiant subspace [Fig. 1(b)] with independent control of $S$ and $M$. By controlling the size of the pseudospin we demonstrate a $g \sqrt{N}$ dependence of the energy exchange rate between the pseudospin and cavity. By varying $M$, we are able to control a delay in the onset of the superradiant emission [25] and we report the first experimental observation of a log dependence of this delay when the pseudospin is near quasiequilibrium at $M=S$. This $\log$ dependence is consistent with predictions from the Tavis-Cummings model. We also observe an abrupt $\pi$-phase shift in the pseudospin microwave emission around this fully inverted state $M=S$. Observations of the $\log$ dependence in the delay and the abrupt phase shift near full pseudospin inversion has eluded previous implementations of strongly coupled spin ensembles due to their much shorter $T_{2}^{*}$ and the low fidelity of their pseudospin rotations (a direct consequence of nonuniform spin-cavity coupling) [26,27].

The isotopically enriched silicon crystal $\left(<50 \mathrm{ppm}{ }^{29} \mathrm{Si}\right)$ we use in these experiments is phosphorus doped with a density of $3.3 \times 10^{15} \mathrm{~cm}^{-3}\left(5.7 \times 10^{13}\right.$ total donors $)$ and is otherwise highly pure (boron density less than $10^{14} \mathrm{~cm}^{-3}$ ) [18]. A tunable distributed Bragg reflector (DBR) laser (Eagleyard EYP-DBR-1080) is used to controllably polarize the phosphorus donor spin ensemble beyond Boltzmann equilibrium by spin-selective optical pumping of the phosphorus donor no-phonon bound exciton transitions [28]. The efficiency of the optical pumping and the resulting steady-state spin polarization $\left(N=N_{\uparrow}-N_{\downarrow}\right)$ are controlled by detuning the laser from resonance with one of the nophonon bound exciton transitions [Fig. 2(a)]. The laser is tunable between 1077-1081 nm (277.2-278.5 THz) and fiber coupled to deliver $\sim 10 \mathrm{~mW}$ of light to the sample. More than $95 \%$ electron spin polarization is achieved after $300 \mathrm{~ms}$ of resonant optical pumping [Fig. 2(a)]. The ${ }^{31} \mathrm{P}$ nuclei are 
(a)
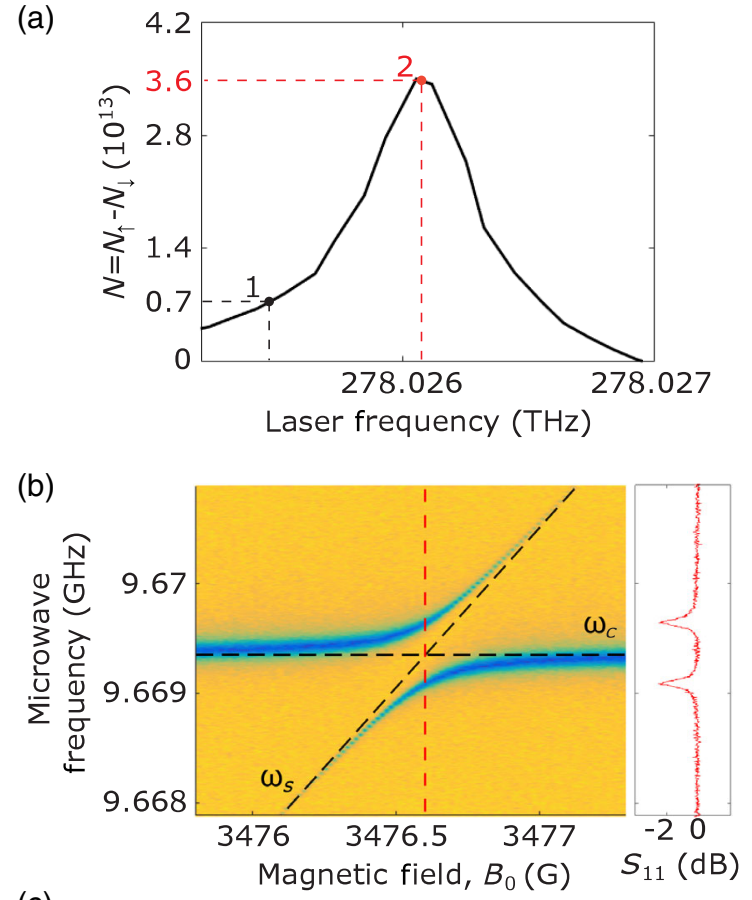

(c)

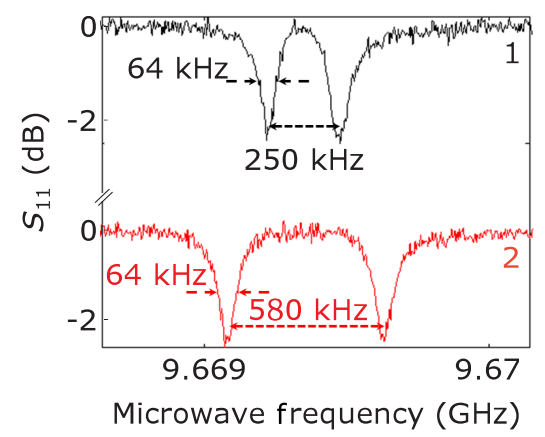

FIG. 2. (a) The number of spins interacting with the resonator $(N)$ is controlled by tuning the laser frequency which is spin-selectively exciting a phosphorus donor no-phonon bound exciton transition [28]. (b) Reflected microwave power $S_{11}$ (color scale) spectrum of the cavity as a function of the spin-ensemble Zeeman splitting $\left(\omega_{s}=g \mu_{B} B_{0}\right)$ as the spin ensemble is brought into resonance with the bare cavity $\left(\omega_{c}\right)$. The clear avoided crossing shows that the system is in the strong-coupling regime with $2 g \sqrt{N}(580 \mathrm{kHz}) \gg \kappa+\gamma(64 \mathrm{kHz})$. (c) The vacuum Rabi splitting $(2 g \sqrt{N})$ of the polariton modes (shown at $\omega_{s}=\omega_{c}$ ) can be controlled by detuning the laser frequency. Curves 1 and 2 were measured while the laser frequency was tuned to points 1 and 2 as indicated in (a).

also polarized to $\approx 25 \%$ during the optical pumping. The mechanism behind this nuclear polarization remains unknown, but it is thought to be due to an enhanced crossrelaxation rate of the donors under illumination [29]. $X$-band $(9.6 \mathrm{GHz})$ ESR experiments are performed with a Bruker ESR spectrometer (Elexsys E580) using a 3D sapphire dielectric resonator (ER-4118X-MD5) in a helium-flow cryostat (Oxford CF935) that is pumped to achieve $1.5 \mathrm{~K}$. A 1.4-T electromagnet (Bruker B-E 25) is used to apply a dc
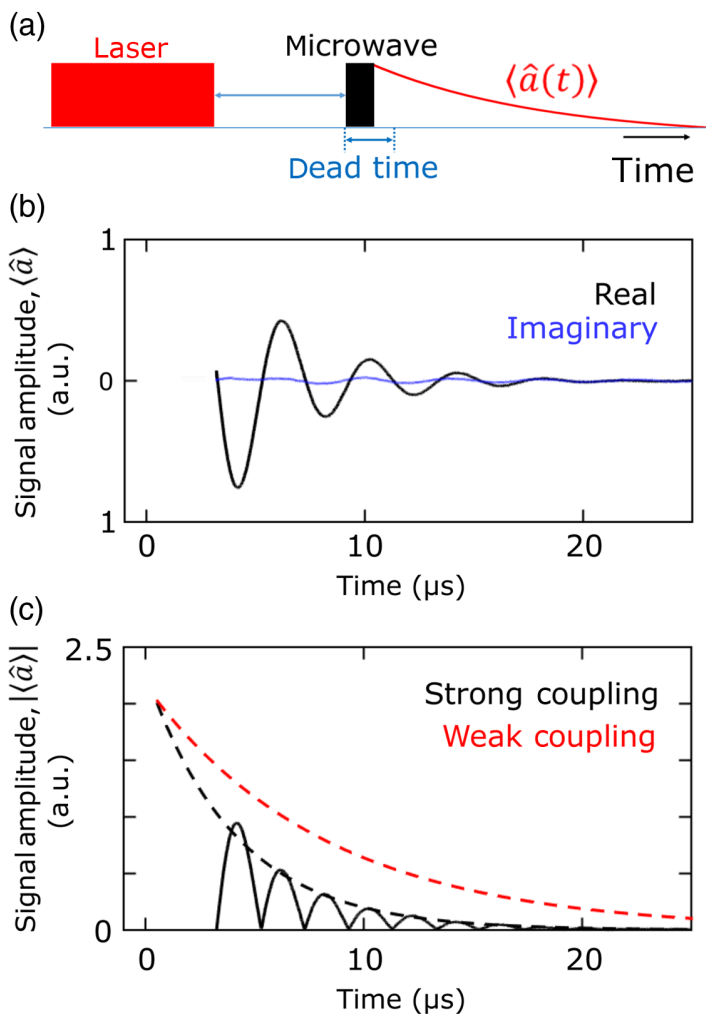

FIG. 3. (a) Pulse sequence of the free-induction decay (FID) experiment. The initial laser pulse sets the number of unpaired spins $N$ and the total spin quantum number $S=N / 2$; the subsequent microwave pulse excites the pseudospin to $|S=N / 2, M\rangle$. The microwave emission from the pseudospin is recorded after the microwave pulse and a 3- $\mu$ s experimental dead time. (b) In-phase $\operatorname{Re}(\hat{a})$ and quadrature $\operatorname{Im}(\hat{a})$ components of the pseudospin microwave emission during the free-induction decay in the strong-coupling regime $\left(M \approx 0, N=3.6 \times 10^{13}\right)$. (c) Amplitude of the microwave emission $|\hat{a}|$ during the FID (black solid line). The FID shows oscillations at rate $g \sqrt{N}=290 \mathrm{kHz}$, many coherent cycles of energy exchange between the spin ensemble and the cavity are resolved. For comparison, the purely exponential decay in the weak-coupling regime is also shown (dashed red line) with $g \sqrt{N}=56 \mathrm{kHz}$, $\kappa=960 \mathrm{kHz}$. The decays are normalized to have the same magnitude at $t=0$.

magnetic field $\left(B_{0}\right)$ to provide a Zeeman splitting to the spin ensemble. A sapphire ring [Fig. 1(a)] is used inside of the copper resonator as a high dielectric material to concentrate the microwave magnetic field $\left(\mathrm{B}_{\mathrm{mw}}\right)$ into the sample volume [30]. In the free-induction decay (FID) experiments, a single 200-ns microwave pulse is used to tip the spins after polarizing with the DBR laser [Fig. 3(a)]. The tipping angle of the microwave pulse is controlled by varying the power of the microwave source (Agilent E8267D).

The stationary states of the system are interrogated by measuring reflected microwave power $\left(S_{11}\right)$ with an Agilent E5071C network analyzer [Figs. 2(b) and 2(c)]. Power saturation is avoided by lowering the probe power to 
$-65 \mathrm{dBm}$ (power incident on the resonator); below this power the measured vacuum Rabi splitting is constant and no power broadening is observed. The $S_{11}$ measurements are performed under continuous illumination from the resonant DBR laser, which, in addition to polarizing the spin ensemble, accelerates spin relaxation $\left(T_{1}\right)$ and reduces power saturation from the microwave probe.

The total ensemble coupling can be determined directly by looking at the eigenfrequencies of the coupled system while the spin ensemble is tuned into resonance with the cavity [Fig. 2(b)]. This technique has been demonstrated for spin ensembles coupled to both 3D volume resonators [31] and 2D superconducting microresonators [32]. The spin transition frequency $\left(\omega_{s}\right)$ is varied through resonance with the cavity $\left(\omega_{c}\right)$ by changing the Zeeman splitting with a magnetic field $\left(B_{0}\right)$, which results in an avoided crossing with a clear splitting showing that the system is in the strong-coupling regime. In particular, with the laser tuned on resonance $\left(N=3.6 \times 10^{13}\right)$ the vacuum Rabi splitting of the polariton modes is $580 \mathrm{kHz}(2 g \sqrt{N})$, which is an order of magnitude larger than their $64-\mathrm{kHz}$ linewidth [Fig. 2(c), curve 2]. The splitting can be controllably reduced by detuning the laser from resonance with the bound exciton transition [curve 1 in Fig. 2(c)]. The large size of the ensemble is important to achieve this large splitting since each spin is weakly coupled to the 3D cavity with a single spin coupling $g=48 \mathrm{mHz}$ [33]. The narrow linewidth of the resonances is defined by combined losses in the cavity ( $\kappa=60 \mathrm{kHz}$ for $Q=75000)$ and dephasing in the spin ensemble $(\gamma=18 \mathrm{kHz}$, measured directly in the weak-coupling regime) [34]. Strong coupling $(2 g \sqrt{N} \gg \kappa+\gamma)$ implies that the system can efficiently and coherently exchange excitations. The cooperativity parameter is $C=2 g^{2} N / \kappa \gamma=91$.

The free evolution of the pseudospin in a cavity is most directly studied in a single-pulse free-induction decay experiment [Fig. 3(a)]. The pseudospin is first tuned into resonance with the cavity $\left(\omega_{s}=\omega_{c}\right)$ and polarized into its ground state $(M=-S)$ with resonant laser pumping thus defining $N=N_{\uparrow}-N_{\downarrow}$ and $S=N / 2$. This is followed by a single resonant microwave pulse $\left(\omega_{\text {drive }}=\omega_{s}=\omega_{c}\right)$ that determines the initial state $(M)$ of the pseudospin before the FID. In the weak-coupling regime the envelope of the freeinduction decay is a simple exponential [Fig. 3(a)] with a characteristic decay time resulting from the inhomogeneous broadening of the spin ensemble $\left(T_{2}^{*}\right)$. However, in the strong-coupling limit we achieve here [Figs. 3(b) and $3(c)$ ], the free-induction decay shows multiple oscillations as energy is coherently exchanged between the spins and the resonator through their coupling at a rate of $2 g \sqrt{N}=$ $580 \mathrm{kHz}$ corresponding to the vacuum Rabi splitting of the polariton modes.

In the strong-coupling regime, the envelope decay of the polariton modes [Fig. 3(c), dashed black line] is faster than the decay in the weak-coupling regime, as it results from a combination of spin dephasing plus resonator losses as the excitations are exchanged between the spin ensemble and the cavity over time. Spin relaxation $\left(T_{1}\right)$ of phosphorus donors at $1.5 \mathrm{~K}$ is slow and does not enter into the dynamics of the free-induction decay [17]. Even with this faster decay we are able to observe 12 collapses and revivals before the free-induction decay falls below the noise level. An experimental dead time of $3 \mu$ s after the microwave pulse limits us from measuring the beginning of the FID signal. The large signal-to-noise ratio and many oscillations we observe here offer clearly resolved dynamics of the coupled spin-ensemble-cavity evolution.

A theoretical description of this experiment begins with a general model for an ensemble of $N$ spin-1/2 particles interacting with a single-cavity mode:

$$
\begin{aligned}
\hat{H}= & \sum_{i} \omega_{i} \hat{s}_{Z i}+\omega_{c} \hat{a}^{\dagger} \hat{a}+2 \sum_{i} g_{i}\left(\hat{a}^{\dagger}+\hat{a}\right) \hat{s}_{X i} \\
& +\sum_{\langle i j\rangle} \hat{\mathbf{s}}_{\mathbf{i}} \cdot \hat{\mathbf{D}}_{\mathbf{i j}} \cdot \hat{\mathbf{s}}_{\mathbf{j}},
\end{aligned}
$$

where $\hat{a}^{\dagger}, \hat{a}$ are the creation and annihilation operators for the cavity field photons of frequency $\omega_{c}, \hat{s}_{k i}(i=1, \ldots, N$ and $k=X, Y, Z)$ are the single spin $(S=1 / 2)$ matrices for a spin with transition frequency $\omega_{i}$ and spin-cavity coupling $g_{i}$. The last term $\left(\hat{\mathbf{D}}_{\mathbf{i j}}\right)$ describes dipolar interactions between spins.

Direct diagonalization of this Hamiltonian for large spin ensembles is not possible; however, several simplifying approximations can be made to good accuracy. The spin ensemble has a narrow inhomogeneous distribution in Zeeman frequencies compared to the spin-cavity coupling $\left(g \sqrt{N} T_{2}^{*} \gg 1\right)$ so that, for the duration of our FID experiment, we can treat the spins as having identical transition frequencies $\omega_{i} \approx \omega_{s}$. The distribution in the individual spin-cavity coupling $(g)$ is mostly defined by the microwave magnetic field inhomogeneity along the length of the sample $(5 \mathrm{~mm})$. For our volume resonator this variation has been measured to be less than 5\% [35]. Thus, to a good approximation, the spin-ensemble-cavity system is in the small sample limit with a uniform spin-cavity coupling $\left(g_{i} \approx g\right)$ [19,36]. Finally, the dipolar coupling between spins [last term in Eq. (1)] at the $3.3 \times 10^{15} \mathrm{~cm}^{-3}$ donor density used in this experiment is $\sim 100 \mathrm{~Hz}$ and negligible on the 30- $\mu$ s time scale measured here [37]. With both the Zeeman frequency and the cavity coupling being constant across all of the spins, the ensemble can be treated as a single large pseudospin with a collective spin operator $\hat{S}_{(z, \pm)}=\sum_{i} \hat{S}_{(z, \pm) i}$. Including all of these considerations, Eq. (1) reduces to the Tavis-Cummings model,

$$
\hat{H}_{\mathrm{TC}}=\omega_{s} \hat{S}_{Z}+\omega_{c} \hat{a}^{\dagger} \hat{a}+g\left(\hat{a}^{\dagger} \hat{S}_{-}+\hat{a} \hat{S}_{+}\right),
$$

which is a generalization of the Jaynes-Cummings model for a single collective pseudospin $\hat{S}$. From this model we 
derive the equations of motion for the pseudospin-cavity system:

$$
\begin{aligned}
\langle\dot{\hat{a}}(t)\rangle & =-\kappa\langle\hat{a}(t)\rangle-i g\left\langle\hat{S}_{-}(t)\right\rangle-i V(t), \\
\left\langle\dot{\hat{S}}_{-}(t)\right\rangle & =-\gamma\left\langle\hat{S}_{-}(t)\right\rangle+2 i g\langle\hat{a}(t)\rangle\left\langle\hat{S}_{z}(t)\right\rangle \\
\left\langle\dot{\hat{S}}_{z}(t)\right\rangle & =i g\left(\left\langle\hat{a}^{\dagger}(t)\right\rangle\left\langle\hat{S}_{-}(t)\right\rangle-\langle\hat{a}(t)\rangle\left\langle\hat{S}_{-}(t)\right\rangle^{*}\right) .
\end{aligned}
$$

These are the Maxwell-Bloch equations for the expectation values $\langle\hat{a}\rangle,\left\langle\hat{S}_{-}\right\rangle$, and $\left\langle\hat{S}_{z}\right\rangle$ [38]. The dissipation factors, $\kappa$ and $\gamma$, are introduced using the standard master equation formalism for the open system [39]. We also add a classical drive term with amplitude $V(t)$ set by the microwave source. To simplify these equations we take the semiclassical limit by neglecting correlations between the spin-ensemble and spin-cavity photons $\left(\left\langle\hat{a} \hat{S}_{i}\right\rangle=\langle\hat{a}\rangle\left\langle\hat{S}_{i}\right\rangle\right.$ for $i=+,-, z)$. This approximation is valid given the large size of our spin ensemble ( $N \sim 10^{13}$ spins). We use Eq. (3) to simulate the dynamics of the free-induction decay (Fig. 4).

In the absence of losses $(\kappa, \gamma=0)$ the total spin of the ensemble is conserved $\left(\left[\hat{H}_{\mathrm{TC}}, \hat{S}^{2}\right]=0\right)$ and the system evolution is a trajectory on the surface of the Bloch sphere within the superradiant subspace [Fig. 1(b)]. Dephasing from inhomogeneous broadening $\left(T_{2}^{*}\right)$ and cavity photon dissipation $(\kappa)$ open up a channel for mixing with subradiant states so that $S$ is no longer preserved and the system enters states in the interior of the sphere [Fig. 4(b)] [40]. In our experiment this dephasing is not refocused and thus is irreversible, so that it can be taken as a single loss rate $\gamma$ for the transverse magnetization of the spin ensemble. The value for $\gamma$ (also used in the $S_{11}$ measurements) is extracted directly from the free-induction decay in the weak-coupling regime where spin dephasing is the only contribution. Photon losses are also taken into account with a single parameter $\kappa=\kappa_{\text {ext }}+\kappa_{\text {int }}$, which represents both internal loss in the cavity $\left(\kappa_{\text {int }}\right)$ and external loss through the antenna coupling $\left(\kappa_{\text {ext }}\right)$. This parameter is extracted by measuring the linewidth of the cavity resonance under low excitation where strong-coupling effects are negligible. The 64-kHz polariton linewidth in Fig. 2(c) is a combination of both $\kappa$ and $\gamma$, with $\kappa \gg \gamma$.

A simulation of the coupled system according to these equations of motion is compared to the measured curve in Fig. 4(a). Here, we plot the intensity of the microwave emission [cavity photon number, $n=\left\langle\hat{a}^{\dagger} \hat{a}\right\rangle=\left\langle\hat{a}^{\dagger}\right\rangle\langle\hat{a}\rangle+$ $\mathcal{O}(\log N)]$ derived from the microwave amplitude, which is measured directly and plotted in Fig. 3(c). This procedure is valid for a large spin ensemble since $(\log N) / N \ll 1$. The only fitting parameter we use in this simulation is a scaling parameter for the microwave pulse $[V(t)]$. This parameter accounts for the resonator coupling as well as losses in the microwave excitation path. The same scaling parameter is used consistently for all microwave powers set in our experiments. The simulation shows an excellent fit to the

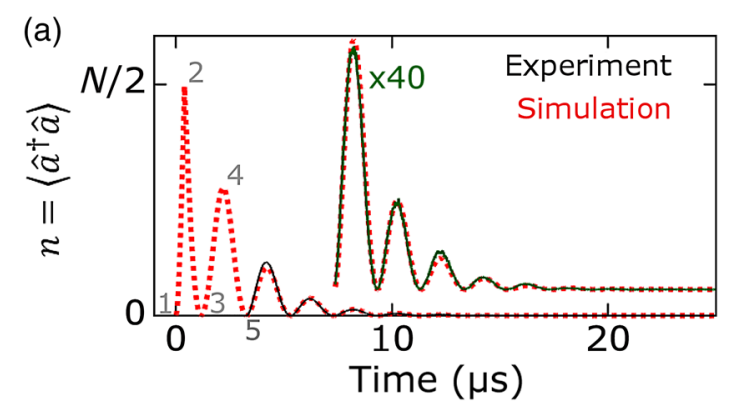

(b)

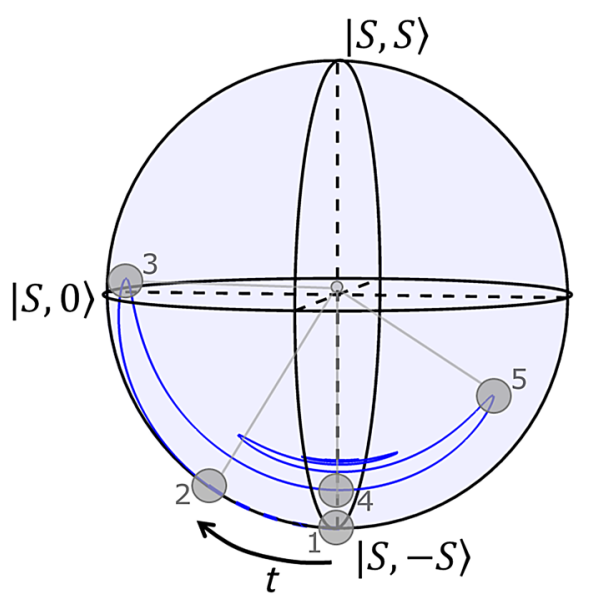

FIG. 4. Collective dynamics between the pseudospin and cavity. (a) Cavity photon number $\left[n(t)=\left\langle\hat{a}^{\dagger} \hat{a}\right\rangle\right]$ during the FID experiment [the square of the FID amplitude shown in Fig. 3(c)] with $N=3.6 \times 10^{13}$ and $M \approx 0$. The simulated fit according to Eq. (3) is also shown (dashed curve). The tail of the decay is magnified $40 \times$ and shown in green. (b) The pseudospin evolution on the Bloch sphere as determined from $\left\langle\hat{S}_{-}(t)\right\rangle$ and $\left\langle\hat{S}_{z}(t)\right\rangle$ in this simulation. The numbers (1-4) on the curves indicate equivalent points in time during the FID on the microwave emission plot (a) and the simulated pseudospin evolution plot (b). The dynamics of the pseudospin in this large $N$ limit is governed by the Maxwell-Bloch equations.

measured FID curve [Fig. 4(a)] with the main difference between the two curves being only the experimental dead time (initial $3 \mu \mathrm{s}$ ). The time evolution of the pseudospin state as determined from this fit is plotted in Fig. 4(b).

The motion of the pseudospin on the Bloch sphere is formally equivalent to a damped pendulum that is kicked into motion by the microwave excitation [20]. The pseudospin is initially polarized to its ground state at the south pole [point 1 on the Bloch sphere in Fig. 4(b)]. A microwave pulse is applied for 200 ns (black arrow), mainly populating the cavity with photons but also starting the initial excitation of the pseudospin (point 2). The driving microwave pulse ends, but the remaining photons in the cavity continue to be transferred to the pseudospin until no photons remain (point 3 on the Bloch sphere). The power of the applied microwave pulse in this example is chosen so that this point would be close to the equator $(|S, 0\rangle)$. This point (3) corresponds to a minimum $(n=0)$ in the 
free-induction decay [Fig. 4(a), point 3] since the photons in the resonator have been fully absorbed by the pseudospin. After reaching this highest point, the excited pseudospin will start to emit photons back into the resonator, reversing the nutation on the Bloch sphere to transition towards its ground state [point 4 in Fig. 4(b)]. During the initial excitation the pseudospin (up until point 3 ) evolves with the same phase as the microwave pulse, but as the pseudospin reverses direction the photons it emits have a phase opposite to that of the initial pulse. The pseudospin eventually fully deexcites to $|S,-S\rangle$ (point 4), releasing all of the photons back into the resonator. This point corresponds to a maximum in the free-induction decay [Fig. 4(a), point 4]. The pseudospin then reabsorbs these photons and is excited to the opposite side of the Bloch sphere (point 5), since the emitted photons are of opposite phase to the initial excitation. This coherent exchange of excitations continues until dissipation and dephasing destroy the ensemble polarization.

Near the maxima and minima of the FID, where $\dot{n}=0$, the pseudospin evolution is dominated by the second derivative (in time) of the cavity photon number $(\ddot{n})$. In the strong-coupling regime (ignoring the dissipation terms) this can be approximated as

$$
\ddot{n} \approx g^{2}\left(\left\langle\hat{S}_{+} \hat{S}_{-}\right\rangle+\left\langle\hat{S}_{-} \hat{S}_{+}\right\rangle\right)+4 g^{2}(n+1 / 2)\left\langle\hat{S}_{z}\right\rangle .
$$

With this expression we can explain the shape of the FID near each extrema. At minima (i.e., point 3 ) there are no photons in the cavity $(n \approx 0)$ and also $\left\langle\hat{S}_{z}\right\rangle=0$; therefore, the second term in Eq. (4) is small. However, the pseudospin is near $|S, 0\rangle$, where correlations between spins lead to superradiant emission with an emission rate that is quadratic in the total number of unpaired spins [1], so that the first term in Eq. (4) is large, $\ddot{n} \propto g^{2}\left\langle\hat{S}_{+} \hat{S}_{-}\right\rangle \propto g^{2} N^{2} / 2$. At maxima (i.e., point 4) the pseudospin is near $|S,-S\rangle$ $\left(\left\langle\hat{S}_{z}\right\rangle \approx-N / 2\right)$, where the correlations between spins in the ensemble are negligible and $\left\langle\hat{S}_{-} \hat{S}_{+}\right\rangle \approx N$ (emission of $N$ uncorrelated emitters). However, most of the photons have transferred back into the cavity $(n \approx N)$, so that the second term in Eq. (4) is now large, resulting in a similarly fast $\ddot{n} \propto-g^{2} N^{2}$. The pseudospin is reexcited by the large population of photons in the resonator $(n \approx N)$, but dissipation and dephasing eventually destroy the ensemble polarization $(N)$, terminating the FID.

The FID we observe is different from superradiance phenomena in free space, where the system is far from the strong-coupling regime. In free space the emitted photons leave the system much faster than they can be reabsorbed by the pseudospin, which means that the second term in Eq. (4) can be neglected. As a result, near $M=-S$ the pseudospin evolves slowly with $\ddot{n} \propto g^{2} N$. This is a factor of $N$ smaller than the value of $\ddot{n}$ we observe in the strongcoupling regime. Additionally, in free space the pseudospin emission dies out entirely after the ground state $|S,-S\rangle$ is

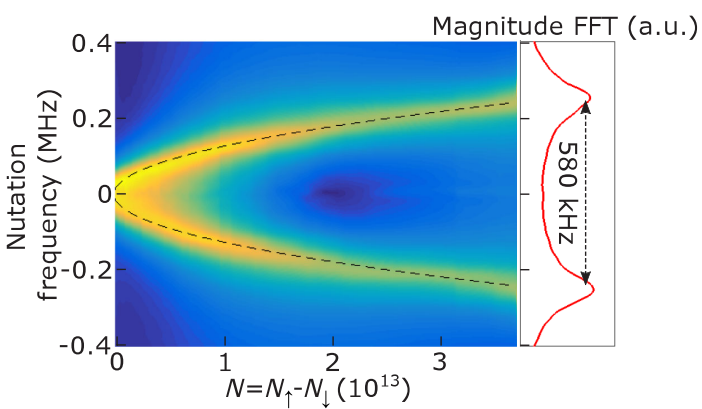

FIG. 5. Fourier transform (plotted as magnitude) of the donor spin-ensemble free-induction decay as a function of the number of unpaired spins in the ensemble $(N)$. The black dashed line shows a $g \sqrt{N}$ dependence of the pseudospin-cavity exchange rate as expected from the Tavis-Cummings model in the large $N$ limit. This gives a single-spin coupling of $g=48 \mathrm{mHz}$ for a uniformly coupled ensemble. The side plot shows a vertical slice at $N=3.6 \times 10^{13} \mathrm{~cm}^{-3}$ for the maximum ensemble coupling that was achieved $(2 g \sqrt{N}=580 \mathrm{kHz})$.

reached because there are no excitations left in the system $(n=0)$ to be reabsorbed by the pseudospin.

In order to better understand the dynamics in this strongcoupling regime, we vary the initial state of the pseudospin to observe its effect on the subsequent evolution. The initial state of the pseudospin $|S, M\rangle$ can be accurately controlled both by adjusting the detuning frequency of the laser, which determines the total spin $S$ [Fig. 2(a)], and by changing the power of the microwave pulse, which determines $M$. The observed dependencies on $S$ and $M$ are plotted in Figs. 5 and 6, respectively.

Varying the laser detuning allows for fine control of the net ensemble polarization $(N)$ and the total spin-ensemblecavity coupling $(g \sqrt{N})$. The magnitude of the Fourier transform of the free-induction decay (Fig. 5) reveals that the pseudospin-cavity exchange frequency scales as $g \sqrt{N}$ (dashed black), in agreement with the large pseudospin picture. The shapes of the polariton peaks in the Fourier transform of the free-induction decay (side plot in Fig. 5) are less resolved than the shapes obtained by reflection spectroscopy [Fig. 2(c)]. This is due to the fact that during the free-induction decay a coherence, created by the initial microwave pulse, leaks to subradiant states $(S<N / 2)$ on the time scale of $T_{2}^{*}$. This leakage gives rise to a timedependent change of $N$ during the FID and decreases the cavity coupling of the pseudospin, effectively smearing out the peaks in the Fourier transforms. This leakage does not occur in the reflection measurements.

The initial $Z$ projection $(M)$ of the pseudospin is set by the tipping angle (power) of the microwave pulse. This tipping angle [defined as polar angle $\theta$ with respect to $|S,-S\rangle$ in Fig. 1(b)] is varied through several full rotations of the pseudospin, and the resulting free-induction decays (real components) are plotted in Fig. 6 (top) accompanied by Maxwell-Bloch simulations (bottom). Rotation angles 


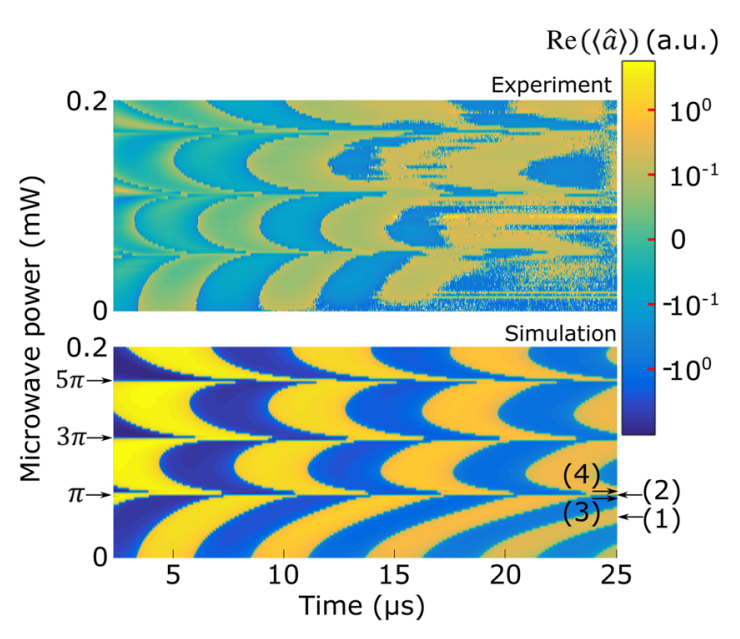

FIG. 6. Top: In-phase component $(\operatorname{Re}\langle\hat{a}\rangle)$ of the pseudospin microwave emission during the FID experiment (time axis) plotted as a function of the rotation angle of the initial microwave pulse (microwave power axis). The signal intensity is plotted using a log color scale in order to emphasize the sign of the signal. The blue (yellow) colors correspond to negative (positive) signal intensities. Bottom: The corresponding FID simulations using the Maxwell-Bloch equations [Eq. (3)]. The rotation angles $(\theta=\pi$, $3 \pi, 5 \pi)$ indicated on the left correspond to the inverted state of the pseudospin $|S, S\rangle$. The numbered arrows (1)-(4) shown on the right correspond to FID experiments shown separately in Fig. 7.

marked as $\pi, 3 \pi$, and $5 \pi$ on the left in Fig. 6 correspond to the pseudospin being fully inverted (near $|S, S\rangle$ ) after photons from the microwave pulse are fully absorbed by the pseudospin. We observe that the period of the FID oscillations does not depend on the rotation angle (initial value of $M$ ) and is always $g \sqrt{N}$. However, there is an overall phase delay in the onset of the oscillations when approaching the fully inverted points at the top of the Bloch sphere (Fig. 6). This shift is more clearly seen when comparing the FID traces in Figs. 7(a) and 7(b) for $\theta \approx \pi / 2$ and $\theta \approx \pi$, respectively.

This delay is explained by considering the pseudospin evolution, starting from $|S, M\rangle$, as an avalanche process with the pseudospin transitioning down the ladder of states in the superradiant subspace towards its ground state [Fig. 1(b)]. Initially there are no photons in the cavity and the emission rate is slow. As the pseudospin deexcites, a larger population of photons builds up in the cavity and the pseudospin emission becomes stimulated. The total time for this process to occur is dominated by the slow time it takes to emit the first few photons in the avalanche, which is longest near full inversion [Fig. 7(b)], since the transition probability of the pseudospin is the smallest there [1]. We use the expression derived in Ref. [2],

$$
\begin{aligned}
t_{D}(M) & \approx \frac{2}{\Gamma}\left[\frac{1}{S-M+1}+\cdots+\frac{1}{N}\right] \approx \frac{2}{\Gamma} \log \frac{2 S}{S-M+1} \\
& =\frac{2}{\Gamma} \log \frac{2}{(1+\cos \theta)},
\end{aligned}
$$
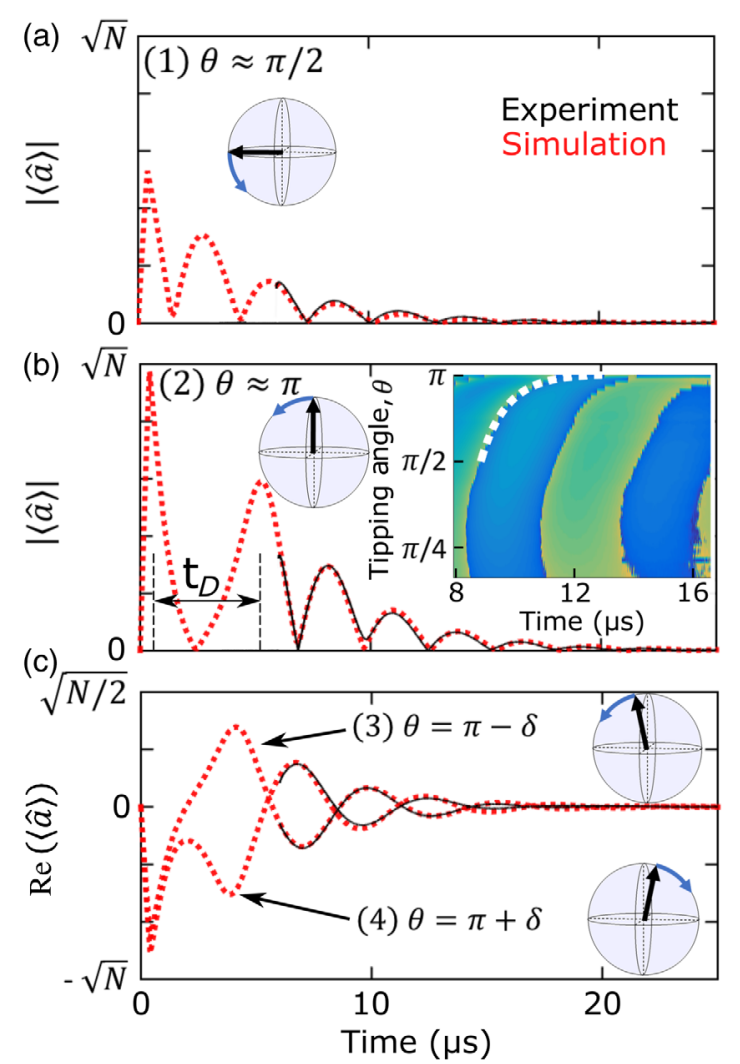

FIG. 7. Comparison of individual FID traces taken as horizontal slices from Fig. 6 for selected rotation angles $(\theta)$ of the microwave pulse. The initial pseudospin state is illustrated on the Bloch sphere for each case (inset). (a),(b) Traces (1) and (2) correspond to rotation angles of $\theta \approx \pi / 2$ (initial pseudospin state $|S, 0\rangle$ ) and $\theta \approx \pi$ (initial pseudospin state $|S, S\rangle$ ), respectively. All oscillations in trace (1) occur at a rate close to $g \sqrt{N}$. In contrast, trace (2) evolves more slowly with a long delay $\left(t_{D}\right)$ in the FID oscillations occurring at the first minimum where the pseudospin reaches full inversion. The rest of the FID trace oscillates at $g \sqrt{N}$ so that this initial delay shows up as an overall phase shift. Inset in (b): A zoomed-in part of the two-dimensional FID plot from Fig. 6 with a fit (white dashed curve) to $t_{D}(M)$ according to Eq. (5). (c) Traces (3) and (4) correspond to slight under- or overrotation with respect to the fully inverted state of the pseudospin $(\theta \approx \pi \pm \delta$, with $\delta=9 \mathrm{deg})$. The phases of the microwave emission are opposite in these two traces since the pseudospin precession reverses direction in the underrotated case but continues in the same direction in the overrotated case.

to fit the delay observed in our experiment [white dashed line in inset of Fig. 7(a)]. We assume an initial state $|S, M\rangle$ with $M \approx S . \Gamma$ is the total spontaneous emission rate of $N$ independent spins (including Purcell enhancement). We extract $\Gamma=2.9 \mathrm{MHz}$ from this fit, which is close to the rate calculated directly from the measured coupling and loss parameters $\left(\Gamma=4 g^{2} N / \kappa=3.2 \mathrm{MHz}\right)$. The longest delay we are able to achieve is $t_{D} \approx 3.5 \mu \mathrm{s}$, corresponding to an initial $Z$ projection of $M=1.77 \times 10^{13}$ and to the tipping angle $\theta=171^{\circ}$. The theoretical limit of the time delay for 
our system, assuming a perfect $\theta=180^{\circ}$ rotation, is $t_{D}(M=S)=2 \log N / \Gamma=21 \mu \mathrm{s}$. This theoretical limit is difficult to achieve experimentally since near full inversion the delay becomes sensitive to small deviations of the rotation angle. Even though our system is very uniform, the delay we obtain is limited by the $5 \%$ variation in cavity coupling across the sample, the $20-\mathrm{mG}$ variation of the static magnietc field across the sample $\left(\delta B_{0} / B_{1}=6 \%\right)$, and lowfrequency fluctuations in the $B_{0}$ field $(\sim 20 \mathrm{mG})$ over a time scale of seconds.

The oscillation delay is also explained by Eq. (4). With the pseudospin near $M=S\left(\left\langle\hat{S}_{z}\right\rangle \approx N\right)$ and no photons in the cavity $(n \ll N)$, the second term is linear in $N$. There are also no correlations between spins here $\left(\left\langle\hat{S}_{+} \hat{S}_{-}\right\rangle \approx N\right)$, so that the first term is also linear in $N$, giving a slow total evolution $\left(\ddot{n} \propto g^{2} N\right)$ as compared to the other extrema in the FID $\left(\ddot{n} \propto g^{2} N^{2}\right)$.

The phase of the microwave emission during the freeinduction decay shifts abruptly by $\pi$ as the pseudospin state is varied through the quasiequilibrium at full inversion around $\theta=\pi, 3 \pi$, and $5 \pi$ in Fig. 6 . This phase shift is more clearly seen in Fig. 7(c) where two traces are shown that correspond to slight underrotation (trace 3 ) and slight overrotation (trace 4) of the pseudospin relative to full inversion. This phase shift is explained by looking at the evolution of the pseudospin on the Bloch sphere. When the microwave drive excites the pseudospin short of the fully inverted state $[\theta=\pi-\delta$, trace 3 in Fig. 7(c)], just after all of the cavity photons have been absorbed, the pseudospin reverses directions on the Bloch sphere in order to evolve towards its ground state. The phase of the emitted photons in this case has an opposite phase to the original photons from the microwave drive. On the other hand, when the cavity photons oversaturate the pseudospin $[\theta=\pi+\delta$, trace 4 in Fig. 7(c)] and drive it past the fully inverted state, then the pseudospin will continue to evolve in the same direction as it transitions towards its ground state. In this case the emitted photons are of the same phase as the initial microwave drive-opposite to the phase of the pseudospin emission in the underrotated experiment.

The quasiequilibrium we observe when the pseudospin is at full inversion is a clear indication that the full dynamics of the Maxwell-Bloch equations must be included to model our system. This is different from the more extensively studied low-excitation limit where the pseudospin is always near $M=-S$, and to good approximation $\hat{S}_{z}$ can be considered to be a constant of motion (HolsteinPrimakoff approximation) [26,41-43], With this approximation the equations of motion are analogous to a system of two coupled linear oscillators and cannot reproduce the behavior we observe near full inversion. In the lowexcitation limit, the maxima and minima of the FID both evolve with $\ddot{n} \propto g^{2} N$, which is much slower than the $\ddot{n} \propto g^{2} N^{2}$ behavior we observe here.
In conclusion, we demonstrate a strongly coupled spin ensemble with uniform spin-cavity coupling and small inhomogeneous broadening allowing the system to be modeled very accurately as a single large pseudospin. This pseudospin evolves according to the TavisCummings model in the large $N$ limit. The use of highly enriched ${ }^{28} \mathrm{Si}$ gives a small inhomogeneous broadening (long $T_{2}^{*}$ ) providing a wide window for viewing the collective pseudospin-cavity dynamics. In particular, we are able to observe the pseudospin-cavity dynamics with a large number of excitations where the Holstein-Primakoff approximation is no longer valid. We can prepare an arbitrary initial state of the pseudospin $|S, M\rangle$ through a combination of optical polarization (determining $S$ ) and microwave manipulation (determining $M$ ). From this we observe the coherent exchange of energy between the pseudospin and the cavity at a rate $g \sqrt{N}$, being independent of $M$. Control over $M$ allows us to view, for the first time, the pseudospin dynamics as it approaches a quasiequilibrium near full inversion. In particular, we observe (and can control) a delay in the pseudospin microwave emission when it is near the quasiequilibrium point. We find that this delay scales with the initial state of the pseudospin $(|S, M\rangle)$ as $\log [2 S /(S-M+1)]$, which is consistent with an expression derived from the Tavis-Cummings model. We also observe that the microwave emission has an abrupt $\pi$-phase shift when the pseudospin is at the quasiequilibrium, which is explained by considering the motion of the pseudospin on the Bloch sphere. This level of pseudospin control is unprecedented and is vital for quantum memory applications. These observations offer novel validations of the TavisCummings model and show that donors in ${ }^{28} \mathrm{Si}$ are a promising platform for implementing a spin-ensemble quantum memory.

Integrating a superconducting qubit inside of a 3D cavity together with the spin ensemble is an appealing next step, and similar hybrid architectures are currently under investigation [23]. Such a hybrid implementation with phosphorus donor electron spins could be challenging due to the large magnetic fields that are required, which could drive the Josephson junctions of the superconducting qubit normal, although junctions have been shown to operate successfully in fields up to $7 \mathrm{~T}$ [44]. One alternative is to utilize a different donor, like bismuth, with a large enough zero-field splitting to enable low-field measurements [45].

This work was supported by the NSF and EPSRC through the Materials World Network and NSF MRSEC Programs (Grants No. DMR-1107606, No. EP/I035536/1, and No. DMR-01420541), and the ARO (Grant No. W911NF-13-1-0179). M. L. W. T. was supported by the Natural Sciences and Engineering Research Council of Canada (NSERC). The work at Keio is supported by 
KAKENHI (S) No. 26220602 and JSPS Core-to-Core Program. The authors extend special thanks to Hakan Türeci and Jonathan Keeling for helpful discussions of the Tavis-Cummings model.

[1] R. H. Dicke, Coherence in Spontaneous Radiation Processes, Phys. Rev. 93, 99 (1954).

[2] M. Gross and S. Haroche, Superradiance: An Essay on the Theory of Collective Spontaneous Emission, Phys. Rep. 93, 301 (1982).

[3] S. Haroche and J. Raimond, Radiative Properties of Rydberg States in Resonant Cavities, Adv. At., Mol., Opt. Phys. 20, 347 (1985).

[4] N. Skribanowitz, I. P. Herman, J. C. MacGillivray, and M. S. Feld, Observation of Dicke Superradiance in Optically Pumped HF Gas, Phys. Rev. Lett. 30, 309 (1973).

[5] M. Gross, C. Fabre, P. Pillet, and S. Haroche, Observation of Near-Infrared Dicke Superradiance on Cascading Transitions in Atomic Sodium, Phys. Rev. Lett. 36, 1035 (1976).

[6] M. Scheibner, T. Schmidt, L. Worschech, A. Forchel, G. Bacher, T. Passow, and D. Hommel, Superradiance of Quantum Dots, Nat. Phys. 3, 106 (2007).

[7] W. Chalupczak and P. Josephs-Franks, Alkali-Metal Spin Maser, Phys. Rev. Lett. 115, 033004 (2015).

[8] J. A. Mlynek, A. A. Abdumalikov, C. Eichler, and A. Wallraff, Observation of Dicke Superradiance for Two Artificial Atoms in a Cavity with High Decay Rate, Nat. Commun. 5, 5186 (2014).

[9] A. F. van Loo, A. Fedorov, K. Lalumière, B. C. Sanders, A. Blais, and A. Wallraff, Photon-Mediated Interactions between Distant Artificial Atoms, Science 342, 1494 (2013).

[10] M. A. Norcia, M. N. Winchester, J. R. K. Cline, and J. K. Thompson, Superradiance on the Millihertz Linewidth Strontium Clock Transition, Sci. Adv. 2, e1601231 (2016).

[11] Y. Zhu, D. J. Gauthier, S. E. Morin, Q. Wu, H. J. Carmichael, and T. W. Mossberg, Vacuum Rabi Splitting as a Feature of Linear-Dispersion Theory: Analysis and Experimental Observations, Phys. Rev. Lett. 64, 2499 (1990).

[12] C. Hettich, C. Schmitt, J. Zitzmann, S. Kühn, I. Gerhardt, and V. Sandoghdar, Nanometer Resolution and Coherent Optical Dipole Coupling of Two Individual Molecules, Science 298, 385 (2002).

[13] K. Baumann, C. Guerlin, F. Brennecke, and T. Esslinger, Dicke Quantum Phase Transition with a Superfluid Gas in an Optical Cavity, Nature (London) 464, 1301 (2010).

[14] I. Diniz, S. Portolan, R. Ferreira, J. M. Gérard, P. Bertet, and A. Auffèves, Strongly Coupling a Cavity to Inhomogeneous Ensembles of Emitters: Potential for Long-Lived Solid-State Quantum Memories, Phys. Rev. A 84, 063810 (2011).

[15] S. L. Mielke, G. T. Foster, J. Gripp, and L. A. Orozco, Time Response of a Coupled Atoms-Cavity System, Opt. Lett. 22, 325 (1997).

[16] M. Brune, F. Schmidt-Kaler, A. Maali, J. Dreyer, E. Hagley, J. M. Raimond, and S. Haroche, Quantum Rabi Oscillation: A Direct Test of Field Quantization in a Cavity, Phys. Rev. Lett. 76, 1800 (1996).
[17] A. M. Tyryshkin, S. Tojo, J. J. L. Morton, H. Riemann, N. V. Abrosimov, P. Becker, H.-J. Pohl, T. Schenkel, M. L. W. Thewalt, K. M. Itoh, and S. A. Lyon, Electron Spin Coherence Exceeding Seconds in High-Purity Silicon, Nat. Mater. 11, 143 (2012).

[18] P. Becker, H.-J. Pohl, H. Riemann, and N. Abrosimov, Enrichment of Silicon for a Better Kilogram, Phys. Status Solidi A 207, 49 (2010).

[19] M. Tavis and F.W. Cummings, Exact Solution for an N-Molecule-Radiation-Field Hamiltonian, Phys. Rev. 170, 379 (1968).

[20] Y. Kaluzny, P. Goy, M. Gross, J. M. Raimond, and S. Haroche, Observation of Self-Induced Rabi Oscillations in Two-Level Atoms Excited Inside a Resonant Cavity: The Ringing Regime of Superradiance, Phys. Rev. Lett. 51, 1175 (1983).

[21] R. J. Thompson, G. Rempe, and H. J. Kimble, Observation of Normal-Mode Splitting for an Atom in an Optical Cavity, Phys. Rev. Lett. 68, 1132 (1992).

[22] Y. Zhu, D. J. Gauthier, S. E. Morin, Q. Wu, H. J. Carmichael, and T. W. Mossberg, Vacuum Rabi Splitting as a Feature of Linear-Dispersion Theory: Analysis and Experimental Observations, Phys. Rev. Lett. 64, 2499 (1990).

[23] G. Kurizki, P. Bertet, Y. Kubo, K. Mlmer, D. Petrosyan, P. Rabl, and J. Schmiedmayer, Quantum Technologies with Hybrid Systems, Proc. Natl. Acad. Sci. U.S.A. 112, 3866 (2015).

[24] C. Grezes, B. Julsgaard, Y. Kubo, M. Stern, T. Umeda, J. Isoya, H. Sumiya, H. Abe, S. Onoda, T. Ohshima, V. Jacques, J. Esteve, D. Vion, D. Esteve, K. Mølmer, and P. Bertet, Multimode Storage and Retrieval of Microwave Fields in a Spin Ensemble, Phys. Rev. X 4, 021049 (2014).

[25] F. Haake, J. W. Haus, H. King, G. Schröder, and R. Glauber, Delay-Time Statistics of Superfluorescent Pulses, Phys. Rev. A 23, 1322 (1981).

[26] S. Putz, D. O. Krimer, R. Amsuss, A. Valookaran, T. Nobauer, J. Schmiedmayer, S. Rotter, and J. Majer, Protecting a Spin Ensemble Against Decoherence in the StrongCoupling Regime of Cavity QED, Nat. Phys. 10, 720 (2014).

[27] Y. Kubo, C. Grezes, A. Dewes, T. Umeda, J. Isoya, H. Sumiya, N. Morishita, H. Abe, S. Onoda, T. Ohshima, V. Jacques, A. Dréau, J.-F. Roch, I. Diniz, A. Auffeves, D. Vion, D. Esteve, and P. Bertet, Hybrid Quantum Circuit with a Superconducting Qubit Coupled to a Spin Ensemble, Phys. Rev. Lett. 107, 220501 (2011).

[28] A. Yang, M. Steger, T. Sekiguchi, M. L. W. Thewalt, T. D. Ladd, K. M. Itoh, H. Riemann, N. V. Abrosimov, P. Becker, and H.-J. Pohl, Simultaneous Subsecond Hyperpolarization of the Nuclear and Electron Spins of Phosphorus in Silicon by Optical Pumping of Exciton Transitions, Phys. Rev. Lett. 102, 257401 (2009).

[29] P. Gumann, O. Patange, C. Ramanathan, H. Haas, O. Moussa, M. L. W. Thewalt, H. Riemann, N. V. Abrosimov, P. Becker, H.-J. Pohl, K. M. Itoh, and D. G. Cory, Inductive Measurement of Optically Hyperpolarized Phosphorous Donor Nuclei in an Isotopically Enriched Silicon-28 Crystal, Phys. Rev. Lett. 113, 267604 (2014).

[30] F. J. Rosenbaum, Dielectric Cavity Resonator for ESR Experiments, Rev. Sci. Instrum. 35, 1550 (1964). 
[31] E. Abe, H. Wu, A. Ardavan, and J. J. L. Morton, Electron Spin Ensemble Strongly Coupled to a Three-Dimensional Microwave Cavity, Appl. Phys. Lett. 98, 251108 (2011).

[32] D. I. Schuster, A. P. Sears, E. Ginossar, L. DiCarlo, L. Frunzio, J. J. L. Morton, H. Wu, G. A. D. Briggs, B. B. Buckley, D. D. Awschalom, and R. J. Schoelkopf, High-Cooperativity Coupling of Electron-Spin Ensembles to Superconducting Cavities, Phys. Rev. Lett. 105, 140501 (2010).

[33] C. Eichler, A. J. Sigillito, S. A. Lyon, and J. R. Petta, Electron Spin Resonance at the Level of $10^{4}$ Spins Using Low Impedance Superconducting Resonators, Phys. Rev. Lett. 118, 037701 (2017).

[34] E. Abe, A. M. Tyryshkin, S. Tojo, J. J. L. Morton, W. M. Witzel, A. Fujimoto, J. W. Ager, E. E. Haller, J. Isoya, S. A. Lyon, M. L. W. Thewalt, and K. M. Itoh, Electron Spin Coherence of Phosphorus Donors in Silicon: Effect of Environmental Nuclei, Phys. Rev. B 82, 121201 (2010).

[35] J. J. L. Morton, A. M. Tyryshkin, A. Ardavan, K. Porfyrakis, S. A. Lyon, and G. A. D. Briggs, Measuring Errors in Single-Qubit Rotations by Pulsed Electron Paramagnetic Resonance, Phys. Rev. A 71, 012332 (2005).

[36] J. M. Raimond, P. Goy, M. Gross, C. Fabre, and S. Haroche, Statistics of Millimeter-Wave Photons Emitted by a RydbergAtom Maser: An Experimental Study of Fluctuations in Single-Mode Superradiance, Phys. Rev. Lett. 49, 1924 (1982).

[37] W. M. Witzel, M. S. Carroll, L. Cywiński, and S. Das Sarma, Quantum Decoherence of the Central Spin in a
Sparse System of Dipolar Coupled Spins, Phys. Rev. B 86, 035452 (2012).

[38] F. Arecchi and R. Bonifacio, Theory of Optical Maser Amplifiers, IEEE J. Quantum Electron. 1, 169 (1965).

[39] V. Tarasov, Quantum Mechanics of Non-Hamiltonian and Dissipative Systems (Elsevier Science, New York, 2008).

[40] V. V. Temnov and U. Woggon, Superradiance and Subradiance in an Inhomogeneously Broadened Ensemble of Two-Level Systems Coupled to a Low-Q Cavity, Phys. Rev. Lett. 95, 243602 (2005).

[41] H. Primakoff and T. Holstein, Many-Body Interactions in Atomic and Nuclear Systems, Phys. Rev. 55, 1218 (1939).

[42] M. G. Raizen, R. J. Thompson, R. J. Brecha, H. J. Kimble, and H. J. Carmichael, Normal-Mode Splitting and Linewidth Averaging for Two-State Atoms in an Optical Cavity, Phys. Rev. Lett. 63, 240 (1989).

[43] J. J. Childs, K. An, M. S. Otteson, R. R. Dasari, and M. S. Feld, Normal-Mode Line Shapes for Atoms in StandingWave Optical Resonators, Phys. Rev. Lett. 77, 2901 (1996).

[44] L. Chen, W. Wernsdorfer, C. Lampropoulos, G. Christou, and I. Chiorescu, On-Chip SQUID Measurements in the Presence of High Magnetic Fields, Nanotechnology 21, 405504 (2010).

[45] P. Haikka, Y. Kubo, A. Bienfait, P. Bertet, and K. Mølmer, Proposal for Detecting a Single Electron Spin in a Microwave Resonator, Phys. Rev. A 95, 022306 (2017). 